\title{
3D printing of molecular models to support undergraduate and graduate teaching and research Marvin Hackert (m.hackert@austin.utexas.edu)
}

Teaching structure/function concepts in chemistry, biochemistry, molecular biology, pharmacy, biomedical engineering, etc. has always been a challenge due to the inherent importance of understanding the role of 3D structure in order to understand function. Most students find it easier to understand the concepts when they have access to molecular models. For protein molecules, the sheer size and number of atoms involved make assembling molecular models from atomic parts impractical. Advances in computers and availability of high quality computer graphics software for the display of molecular models has helped, but does not replace the benefit of being able to hold and examine a physical model.

Three things are needed in order to print a 3D model: 1) a coordinate file, 2) molecular graphics software, and 3) 3D printer. Access to over one million coordinate files from small organic molecules to proteins are available on databases such as the Protein Data Bank (PDB) and the Cambridge Structural Database (CSD). Several high-quality molecular visualization software packages such as Mercury (CSDS) and ChimeraX (UCSD) are available that enable the user to output a stereolithographic (.stl) or other file format suitable for $3 \mathrm{D}$ printing. Finally, access to high quality $3 \mathrm{D}$ printers is becoming common at most universities and public libraries. Users can even avoid steps $1 \& 2$ by visiting sites such as the NIH 3D Print Exchange ( https://3dprint.nih.gov/) that provides access to hundreds of molecular models to download in formats that are already compatible with 3D printers.

Our experience using various 3D printing technologies to generate molecular models for use in the classroom and research laboratories to improve learning will be described. The scale of the model, use of struts and pseudo-bonds, which details to highlight, etc. all factor in to having a robust model suitable for classroom use. Examples of several models will be available. 